\title{
2. Comienzo de la educación superior virtual en Colombia: entre la concentración geográfica de respuestas institucio- nales y el cierre de brechas entre zonas rurales y urbanas
}

\author{
Nicolás Arias-Velandia \\ JAIME GuARnizo-Mosquera \\ DEISY ORTIZ-ROMERO \\ ERIKA GÓMEZ-VILLARREAL \\ LEONARDO ROJAS-BENAVIDES
}

\section{Introducción}

I a educación superior forma personas y de esto se espera que, en retorno, se conviertan en trabajadores calificados y en personas con capacidades para la dirección estratégica y con capacidad de discernimiento crítico basado en conocimiento (Arum y Roksa, 2011; Bogoya, 2006; Celis, Jiménez y Jaramillo, 2013). En una sociedad democrática, es deseable que todos sus miembros tengan acceso a la educación básica y oportunidades equitativas de acceso a la educación superior (Arum y Roksa, 2011; Tomasevsky, 2005).

En concordancia con esto, las oportunidades de acceso a la educación superior se garantizan mediante estrategias colectivas e institucionales de eliminación de barreras a su acceso. Por tanto, es importante analizar la oferta disponible a los estudiantes reales y potenciales de la educación superior y la relación entre estos estudiantes y la cobertura y calidad que logra aquella en las modalidades en las que se ofrece en los años cuando comenzó a haber oferta de programas de educación superior virtual en Colombia.

Nicolás Arias Velandia. Sicólogo por la Universidad Nacional de Colombia y magíster en Educación por la Universidad Pedagógica Nacional. Líder Observatorio de Educación Virtual, Departamento de Investigación, Desarrollo e Innovación, Institución Universitaria Politécnico Grancolombiano. e-mail: nariasv@poligran.edu.co

Jaime Guarnizo Mosquera. Profesional en Estadística por la Universidad Nacional de Colombia y especialista en Finanzas por la Universidad de los Andes. Profesor de la Institución Universitaria Politécnico Grancolombiano. e-mail: jguarnizo@ poligran.edu.co

Deisy Ortiz Romero. Psicóloga por la Institución Universitaria Politécnico Grancolombiano. Miembro del Observatorio de Educación Virtual, Departamento de Investigación, Desarrollo e Innovación, Institución Universitaria Politécnico Grancolombiano. e-mail: dey.ortiz@hotmail.com

Erika Gómez Villarreal. Psicóloga por la Institución Universitaria Politécnico Grancolombiano. Miembro del Observatorio de Educación Virtual, Departamento de Investigación, Desarrollo e Innovación, Institución Universitaria Politécnico Grancolombiano. e-mail: tata010@hotmail.com

Leonardo Rojas Benavides. Psicólogo por la Universidad Nacional de Colombia. Asistente de investigación del Observatorio de Educación Virtual, Departamento de Investigación, Desarrollo e Innovación, Institución Universitaria Politécnico Grancolombiano. e-mail: ramzadkm@gmail.com 
En este capítulo, se analiza el estado de ofertas de educación superior para estudiantes colombianos de la zona rural y de la zona urbana en los primeros años de sus ofertas virtuales, con el análisis de datos y evidencias de diferentes fuentes, con el fin de estimar si se presentaron una situación de brecha de acceso y calidad de educación superior para estudiantes rurales y urbanos, a la luz de la existencia tanto de ofertas presenciales como de ofertas a distancia y virtuales en la educación superior colombiana. Este análisis se basa en la perspectiva de educación basada en la evidencia, que busca el alimento de las prácticas educativas y de las decisiones del sistema educativo según evidencias fiables existentes (Campbell Collaboration, 2006; Hederich, Martínez y Rincón, 2014).

La eliminación de barreras de acceso a la educación superior se opera con la existencia de ofertas de educación con niveles comprobados de calidad y en la relación entre dichas ofertas y la población de potenciales estudiantes de educación superior (Montoya, 2013; Tomasevsky, 2005). La oferta que hacen las instituciones de educación superior en Colombia a sus potenciales estudiantes se realiza, entonces, mediante dos modalidades o metodologías: la presencial, que supone que el estudiante puede asistir a la educación que se le imparte en un espacio físico provisto por la institución, en un tiempo específico y en interacción cara a cara con los profesores y otros estudiantes (Areth, Castro-Martínez y Rodríguez, 2015; Silvio, 2004); y la a distancia, denominada distancia tradicional cuando es mediada por sistemas de contacto con sedes o no basados en tecnología informática en más de 20 \% del trabajo del estudiante durante un periodo, y virtual o distancia virtual, cuando es mediada por recursos informáticos e internet (Montoya, 2013) en 80 \% o más del tiempo del estudiante en actividades académicas durante un periodo.

Según la Unesco, las modalidades de educación a distancia y virtual deben convertirse en instrumentos eficaces para aumentar las oportunidades de acceso a la educación superior a estudiantes que no han podido acceder a esta formación, por no poder recorrer grandes distancias y por no disponer de un solo horario fijo para realizar actividades de clase o dirigidas al aprendizaje (Lugo, Vera y Flood, 2004; Padilla, 2004; Rivera, 2004; Silvio, 2004; Vianney, Torres y Farias, 2004). Sin embargo, Fujita, Krugman y Venables (1999) plantean que en varias sociedades existe una tendencia a concentrar recursos de todo tipo en uno o en pocos puntos específicos del espacio físico y geográfico. De este modo, la posibilidad de acceso en alguno de estos puntos multiplica las opciones de escogencia. Por tanto, y desde esta perspectiva, muchas de las ofertas de bienes y servicios tienen una fuerte tendencia a crecer en los medios y nichos sociales donde estos ya tienen presencia.

En Colombia, esta dinámica parece presentarse en sus brechas entre la zona rural y la zona urbana. Es probable que esto haya surgido históricamente en la tradición consolidada de relaciones entre campo y ciudad o entre zonas rurales y urbanas de América Latina. 
Comunicación, redes, aprendizaje y desarrollo institucional y social

Según Romero (2001), en el modelo de colonización lusoespañol en América se toma como centro de poder la ciudad, que en la península ibérica de fines de la Edad Media era una ciudad centro del poder cristiano señorial, que imponía la segregación a comunidades de musulmanes y judíos. Más adelante, se acentúa la situación porque la ciudad se hace, además de manufacturera y mercantil, industrial, con lo cual se generan migraciones del campo que hacen crecer el número de integrantes de las clases socioeconómicas desfavorecidas en el mundo urbano. En un sentido similar, Colmenares (1987) plantea que durante la Conquista y la Colonia la concentración de propiedad hizo que las ciudades devinieran centros de administración, mientras que los indígenas y otros habitantes se hacían grupos rurales dependientes del poder administrado desde las urbes, con lo cual desarrollaron buena parte de su evolución social en zonas dispersas con economías dependientes de la agricultura y la minería.

En síntesis, en el orden social colombiano ...y al parecer también en otros países de América Latina- existe una brecha consistente en una enorme e inequitativa diferencia de acceso a la educación y a otras oportunidades entre los pobladores de zonas urbanas y rurales. En concordancia con el ideal postulado por la Unesco, este constituye un gran desafío a las ofertas de educación superior, y más específicamente a su oferta en educación virtual y a distancia. Cabe la duda de hasta qué punto logran estas formas de educación dar respuesta eficaz a este desafío en los inicios de la implementación de la educación superior por medios virtuales.

Como parece confirmarse en respuesta a la hipótesis desde los planteamientos de Fujita, Krugman y Venables (1999), la urbanización es un factor que incide en el logro educativo. Los jóvenes de medios urbanos y con mayor acceso a servicios domiciliarios y medios de comunicación como el computador tienden a mostrar mayores niveles de logro educativo que sus pares sin acceso a dichos medios (Hederich y Camargo, 2000; Hederich, 2007). Según Hederich y Camargo (2000), es posible que un mayor involucramiento en los medios que exige la modernización urbana incida en las oportunidades para el desarrollo de conocimientos.

En relación con esto, se presentan mayores puntajes en medidas de logro educativo en pruebas de evaluación censal en estudiantes de poblaciones urbanas que en poblaciones rurales (Hederich y Camargo, 2000), relacionado específicamente con los siguientes factores:

- Nivel socioeconómico de la familia.

- Nivel sociocultural de la familia (indicado por el nivel educativo de ambos padres).

- Características de estructura, extensión y tamaño del grupo familiar. 
Los hallazgos de estos dos autores, si bien reafirman la relación entre nivel socioeconómico y logro educativo, muestran una relación mucho más estrecha entre el nivel sociocultural de la familia y el alto logro educativo evaluado externamente mediante pruebas censales (Hederich y Camargo, 2000; Hederich, 2007). Estos logros parecen también asociarse con la ubicación de la residencia de los estudiantes a núcleos regionales centrales del movimiento urbano y económico -Bogotá y Medellín- y con la residencia en regiones como la región paisa (Antioquia y Antiguo Caldas) y la región norandina de Boyacá y los Santanderes.

Lo anterior nos da una idea más precisa del desafío que enfrentaron las ofertas de educación superior a distancia y virtual en Colombia para cumplir con el cometido de la Unesco en cuento dar acceso a educación superior a grupos poblacionales que habitualmente no han tenido acceso a ella. En este sentido Silvio (2004) comienza a plantear si es realmente el surgimiento de la educación virtual una respuesta a la demanda de educación superior de América Latina.

Según el informe de IESALC-Unesco, la implementación inicial de programas de educación virtual en América Latina estuvo fuertemente condicionada por la disponibilidad de recursos de tecnologías informáticas y de comunicación (Silvio, 2004). Su tendencia mostraba una llegada inicial a grupos de personas que ya tenían formación o por medio de algunos convenios con el mundo empresarial, y solo se ligaba a una actividad más extendida a otros contextos - como el mundo rural- cuando comienza la intervención de los Estados o de los consorcios de universidades oficiales con empresas privadas, como Brasil (Vianney, Torres y Farias, 2004) y Argentina (Lugo, Vera y Flood, 2004). A pesar de esto, en tales países una buena parte de la oferta sigue concentrada en los grupos de ingresos medios y altos, que también han sido los tradicionales beneficiarios de otros tipos de educación (Silvio, 2004; Lugo, Vera y Flood, 2004; Padilla, 2004; Vianney, Torres y Farias, 2004).

Silvio (2004) planteaba, al presentar un informe sobre el inicio de la implementación de la educación virtual en la región, que algunas de las primeras experiencias de implementación se dan entre 1995 y 2000, coincidentes con el inicio de la popularización de la web en el mundo (Prensky, 2001), y ubica como antecedentes directos de esta implementación los programas a distancia tradicionales en Costa Rica, Colombia y Venezuela, que tienen como modelo de su trabajo los desarrollos de la Open University del Reino Unido. En ese sentido, el crecimiento en la educación virtual en la región latinoamericana comenzó acelerado, pero bajo, debido al atraso en tecnología y redes en la región, la falta de interactividad en el diseño de plataformas e interfaces iniciales (centradas en modelos web 1.0), el alto costo de instalación de infraestructura y las resistencias en el mundo académico al reconocimiento de posibilidades de educación virtual (Silvio, 2004). 
En este contexto, este estudio se pregunta ¿cuáles evidencias dan cuenta del avance de la oferta de educación superior a jóvenes colombianos entre 17 y 21 años, utilizando las ofertas de educación presencial, a distancia y virtual, para avanzar en el cierre de brechas de oportunidades educativas entre estudiantes de zonas rurales y urbanas?

\section{Método}

\section{Tipo de investigación}

Este estudio se basa en el paradigma de educación basada en la evidencia (Hederich, Martínez y Rincón, 2014) y consiste en la indagación de fuentes secundarias de datos y evidencias que den cuenta de las posibilidades de acceso y aprendizaje alcanzado en estudiantes colombianos de 17 a 21 años en zonas rurales y urbanas, de la oferta de estudios disponible para estos jóvenes y del avance que se ha reportado institucionalmente para responder a las necesidades de acceso y calidad en educación superior tanto presencial como a distancia tradicional y virtual. Las fuentes de datos y evidencias consultados para su elaboración son:

- Bases de datos de acceso abierto del Departamento Administrativo Nacional de Estadística (DANE).

- Sistema Nacional de Información de la Educación Superior (SNIES).

- Informes históricos consolidados y bases de datos de logros de estudiantes en prueba Saber 11 (de cierre de estudios de educación secundaria y media) del Instituto Colombiano para la Evaluación de la Educación (Icfes).

- Reporte oficial de resultados de la prueba PISA-OCDE 2012 realizada a una muestra representativa de población de estudiantes de 15 años del país.

\section{Procedimiento de indagación}

En primer lugar, se obtuvo acceso a los datos y las evidencias mencionados en el apartado anterior, a través de las páginas web del DANE, del MinEducación y del Icfes. De ellos se extrajo información concerniente a diferencias entre estudiantes del medio rural y urbano de jóvenes entre 17 y 21 años y de las ofertas de educación superior para sus potenciales estudiantes. Luego, se hizo agrupación de estos datos en torno a tres ejes de la información: entorno de los estudiantes potenciales de educación superior en Colombia (condiciones poblacionales de los estudiantes colombianos entre 17 y 21 años), respuesta institucional a las condiciones de estudiantes reales y potenciales de la educación superior en 
Colombia (condiciones de la oferta educativa existente de educación superior presencial, a distancia y tradicional y a distancia virtual) y logros de estudiantes colombianos en zonas rurales y urbanas que podrían acceder a la educación superior (reporte del tipo de logro educativo evaluado por pruebas externas que da cuenta de lo aprendido por estudiantes para poder tener mayores oportunidades y aprovechamiento de las ofertas de educación superior en Colombia).

De esta información organizada se tomaron datos directos generados por las entidades, se construyeron figuras o tablas propias basadas en la información dada por las entidades y se realizó un reporte de indagación alternando explicaciones y evidencias.

\section{Procedimiento de análisis de la información}

De la información obtenida se procuró mantenerla en datos descriptivos o en el original que manejaban los reportes y las fuentes descritos en los dos apartados anteriores, mostrándolos como evidencia de la explicación verbal que se desarrolla en los resultados. Sin embargo, para la presentación de la información, se realizaron algunas transformaciones indicadas en la tabla 2.1.

\section{Limitaciones de la indagación realizada}

Este estudio tiene dos limitaciones: se basa en información de organismos que no tenían actualización permanente (al menos anual) y los datos sobre educación virtual y a distancia no necesariamente se ubican en el lugar de las instituciones de origen, con lo cual no se cuenta todavía para este estudio con información más concreta sobre los datos de procedencia de los estudiantes.

Por otra parte, esta indagación se inscribe en evidencias de un periodo entre 2002 y 2014, por lo cual hace un recuento histórico relacionado con el tiempo de la primera inscripción oficial de programas de educación superior virtual en Colombia ante el MinEducación (entre 2006 y 2010), y no refleja necesariamente el panorama más actual del tema en Colombia. Se concentra en las tendencias de los años iniciales de implementación de dichos programas en modalidad o metodología virtual.

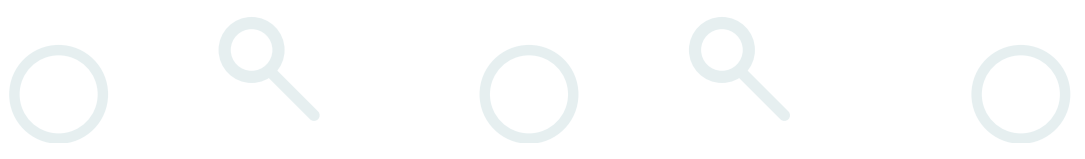


Comunicación, redes, aprendizaje y desarrollo institucional y social

Tabla 2.1. Información extraía, transformaciones y propósitos de las fuentes de información usadas

\begin{tabular}{|c|c|c|}
\hline $\begin{array}{l}\text { Fuente de } \\
\text { información }\end{array}$ & Transformación de los datos de la fuente & Propósito \\
\hline $\begin{array}{l}\text { Base de datos } \\
\text { del DANE }\end{array}$ & $\begin{array}{l}\text { Cálculo de proporción obtenido mediante el } \\
\text { cociente de la división entre número de es- } \\
\text { tudiantes en zona rural y zona urbana, según } \\
\text { datos primarios del DANE }\end{array}$ & $\begin{array}{l}\text { Mostrar una mayor o menos des- } \\
\text { proporción de acceso a educación } \\
\text { entre estudiantes de zonas rurales } \\
\text { o urbanas de Colombia }\end{array}$ \\
\hline $\begin{array}{l}\text { Base de datos } \\
\text { del DANE }\end{array}$ & $\begin{array}{l}\text { Generación de una tabla de los departamen- } \\
\text { tos de Colombia con mayor desproporción } \\
\text { de acceso de estudiantes a la educación se- } \\
\text { cundaria y media y a la educación de jóvenes } \\
\text { y adultos }\end{array}$ & $\begin{array}{l}\text { Mostrar departamentos con mayor } \\
\text { desproporción en ambos grupos } \\
\text { poblacionales }\end{array}$ \\
\hline $\begin{array}{l}\text { Base de datos } \\
\text { del DANE }\end{array}$ & $\begin{array}{l}\text { Cálculo del porcentaje aportado por Bogotá } \\
\text { a la población nacional de estudiantes entre } \\
17 \text { y } 21 \text { años }\end{array}$ & $\begin{array}{l}\text { Tener una base de la población que } \\
\text { potencialmente accederá a la edu- } \\
\text { cación superior de pregrado tanto } \\
\text { en Bogotá como fuera de ella }\end{array}$ \\
\hline $\begin{array}{l}\text { Base de datos } \\
\text { del SNIES }\end{array}$ & $\begin{array}{l}\text { Cálculo de la cobertura lograda en educación } \\
\text { superior en Bogotá y en el resto del país sin } \\
\text { considerar esta ciudad }\end{array}$ & $\begin{array}{l}\text { Calcular el tipo de oferta de educa- } \\
\text { ción superior diferenciando Bogotá } \\
\text { y el resto de Colombia }\end{array}$ \\
\hline $\begin{array}{l}\text { Reportes de } \\
\text { resultados del } \\
\text { Icfes }\end{array}$ & $\begin{array}{l}\text { Reporte de datos de estudiantes en prueba } \\
\text { Icfes-Saber } 11 \text { (al finalizar educación secun- } \\
\text { daria y media) }\end{array}$ & $\begin{array}{l}\text { Realizar análisis comparativos del } \\
\text { logro educativo (aprendizaje alcan- } \\
\text { zado) de estudiantes de ambas zo- } \\
\text { nas }\end{array}$ \\
\hline $\begin{array}{l}\text { Reportes de } \\
\text { resultados de } \\
\text { estudiantes } \\
\text { colombianos } \\
\text { en evaluación } \\
\text { internacional } \\
\text { PISA-OCDE }\end{array}$ & $\begin{array}{l}\text { Extracción de resultados en pruebas PI- } \\
\text { SA-OCDE de los estudiantes colombianos di- } \\
\text { ferenciando los puntajes de aquellos proce- } \\
\text { dentes de zonas rurales y de zonas urbanas }\end{array}$ & $\begin{array}{l}\text { Realizar análisis comparativos del } \\
\text { logro educativo (aprendizaje alcan- } \\
\text { zado) de estudiantes de ambas zo- } \\
\text { nas }\end{array}$ \\
\hline
\end{tabular}

Fuente: elaboración propia.

\section{Resultados}

\section{El entorno de los estudiantes potenciales de la educación superior en Colombia}

De acuerdo con los datos del Censo General 2005 (DANE, 2011), la proporción alumnos en zona urbana/alumnos en zona rural se hace mayor en el nivel de educación secundaria y media (4.5235), y desciende un poco en jóvenes y adultos (3.8309). Este índice proporcional es menor 
en el nivel de básica primaria (2.196885). Los departamentos con proporción urbano/rural de 5 a 1 o mayor en educación secundaria y media y en jóvenes y adultos se muestran en la tabla 2.2.

Tabla 2.2. Acceso a educación en departamentos de Colombia con proporción 5/1 entre habitantes urbanos y rurales

\begin{tabular}{l|l}
\hline Departamento & Grupo poblacional con proporción 5/1 en urbano/rural \\
\hline Amazonas & Jóvenes y adultos \\
\hline Antioquia & Secundaria y media, jóvenes y adultos \\
\hline Atlántico & Secundaria y media, jóvenes y adultos \\
\hline Bogotá & Secundaria y media, jóvenes y adultos \\
\hline Casanare & Secundaria y media, jóvenes y adultos \\
\hline Cauca & Jóvenes y adultos \\
\hline Guajira & Secundaria y media \\
\hline Magdalena & Secundaria y media \\
\hline Meta & Secundaria y media \\
\hline Norte de Santander & Secundaria y media, jóvenes y adultos \\
\hline Quindío & Secundaria y media, jóvenes y adultos \\
\hline Risaralda & Secundaria y media \\
\hline Santander & Secundaria y media \\
\hline Valle del Cauca & Secundaria y media, jóvenes y adultos \\
\hline Vaupés & Jóvenes y adultos \\
\hline
\end{tabular}

Fuente: DANE (2011).

Este análisis por departamentos nos muestra entonces lo siguiente:

- Una mayor desproporción de matrículas en secundaria y media solo entre estudiantes de zona urbana y rural en favor de los primeros en La Guajira, Magdalena, Meta, Risaralda y Santander. Según la misma fuente, Risaralda y Santander se cuentan entre los departamentos con mayor población, y La Guajira y Magdalena como dos departamentos con un alto porcentaje de población indígena, lo cual indica diferencias de acceso muy grandes entre zonas rurales y urbanas para un gran número de jóvenes, que afecta probablemente a varios grupos indígenas.

- Una mayor desproporción de matrículas en jóvenes y adultos solo entre estudiantes de zona urbana y rural en favor de los primeros en Amazonas, Cauca y Vaupés. Estos tres departamentos cuentan también con un importante número de personas pertenecientes a grupos indigenas, donde dos de ellos (Amazonas y Vaupés) se ubican en 
Comunicación, redes, aprendizaje y desarrollo institucional y social

la región de la selva amazónica y por ello cuentan con pocas vías de acceso y comunicación desde las regiones centrales del país.

- Una mayor desproporción de matrículas entre estudiantes en secundaria y media, y también en jóvenes y adultos, de zona urbana y rural, en favor de los primeros en Antioquia, Atlántico, Bogotá, Casanare, Norte de Santander, Quindío y Valle de Cauca. Esto muestra una alta probabilidad de diferencias de acceso tanto a la educación secundaria y media como a las ofertas de educación superior por parte de un alto volumen de la población joven colombiana, ya que en este grupo se encuentran los departamentos y distritos que concentran el mayor número de pobladores del país (Antioquia, Atlántico, Bogotá, Norte de Santander, Quindío y Valle de Cauca).

- Cabe anotar que donde más se da la mencionada desproporción de acceso a educación entre estudiantes de zonas rurales y de zonas urbanas es en departamentos con mayor tradición de producción económica y con mejores vías de acceso y comunicación: tal era el caso de Antioquia, Atlántico, Bogotá, Norte de Santander, Quindío, Risaralda, Santander y Valle del Cauca.

Del mismo modo, en, capital del país, la población entre 17 y 21 años aumenta constantemente y con ella sus demandas de ofertas de educación. En 2009, esta población fue casi la cuarta parte de la población total nacional en esta franja de edad. La población colombiana muestra un crecimiento sostenido de 2002 a 2009, mientras que la población de Bogotá en este mismo periodo señala una tendencia al aumento y a una leve disminución hacia el final de dicho periodo (figura 2.1).

Figura 2.1. Número de pobladores entre 17 y 21 años en Colombia y en Bogotá.

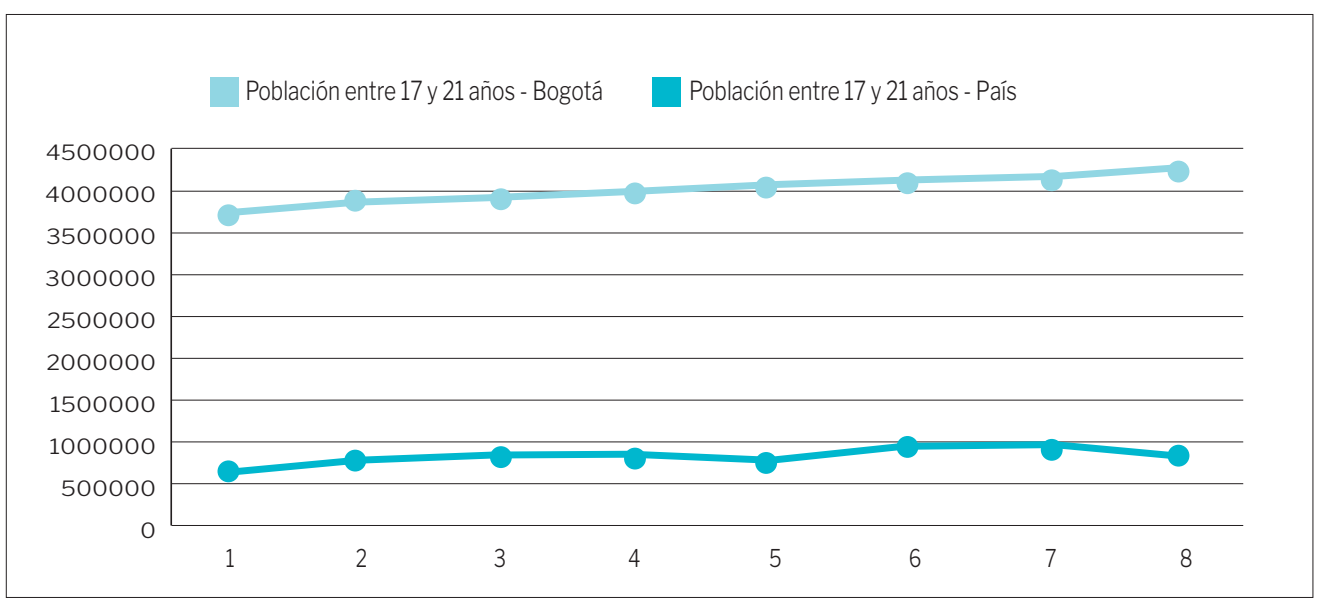

Fuente: DANE (2011). 
Por tanto, el porcentaje que Bogotá aporta a la población del país aumentó constantemente de 16.88 \% en 2002 a 23.06 \% en 2009 (DANE, 2011; Institución Universitaria Politécnico Grancolombiano, inédito) (figura 2.2).

Figura 2.2. Número de pobladores entre 17 y 21 años en Colombia y en Bogotá y porcentaje aportado por Bogotá a esta población nacional.

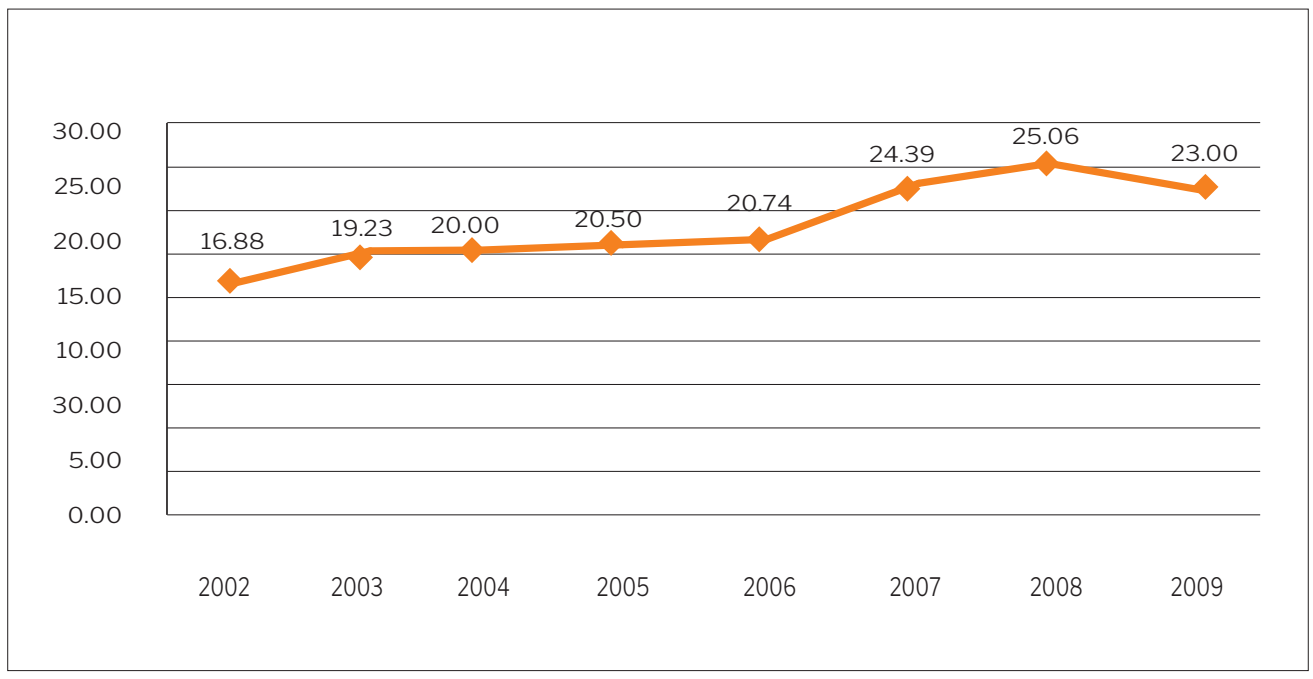

Fuente: DANE (2011).

Lo anterior muestra que el acceso a la educación no se estaba cumpliendo a cabalidad, incluso, desde niveles anteriores a la educación superior para una gran cantidad de jóvenes del país, lo cual hacía difícil poder cumplir, al menos hasta 2014, el cometido de la Unesco de dar acceso a poblaciones como la rural en el acceso a educación superior. A continuación, se ilustra este panorama en las respuestas que la oferta de instituciones de educación superior estaba dando a este desafío.

La respuesta institucional a las condiciones de estudiantes reales y potenciales de la educación superior en Colombia

Ante el panorama anterior cabe preguntarse, ¿cuál ha sido la respuesta de las ofertas de educación superior? La respuesta en cifras del MinEducación indica que, para 2014, el número de programas presenciales sigue siendo mucho mayor que los programas a distancia tradicional y a distancia virtual: es una proporción casi de diez programas presenciales por un programa a distancia presencial y virtual (tabla 2.3). 
Tabla 2.3. Porcentaje de programas en Colombia y en Bogotá por niveles de formación y por modalidades de oferta de educación superior en 2014

\begin{tabular}{|c|c|c|c|c|c|c|c|c|c|c|}
\hline $\begin{array}{l}\text { Nivel de } \\
\text { formación }\end{array}$ & Técnic & & & Tecnól & & & $\begin{array}{l}\text { Pro } \\
\text { unis }\end{array}$ & $\begin{array}{l}\text { sional } \\
\text { rsitario }\end{array}$ & & TOTAL \\
\hline 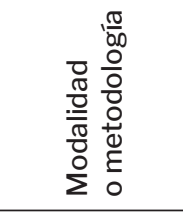 & 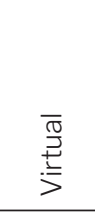 & 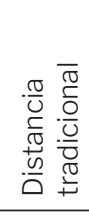 & $\begin{array}{l}\frac{\bar{\sigma}}{0} \\
\frac{0}{0} \\
0 \\
0 \\
\stackrel{0}{0}\end{array}$ & 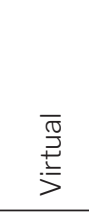 & 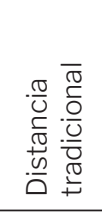 & 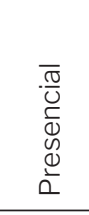 & $\stackrel{\frac{\pi}{3}}{\stackrel{5}{>}}$ & $\begin{array}{l}\frac{1}{0} \\
\frac{\pi}{5} \\
\frac{\pi}{0} \\
\frac{\pi}{0} \\
\frac{\pi}{\pi} \\
\frac{.0}{0} \\
\frac{0}{0} \\
\frac{0}{4}\end{array}$ & 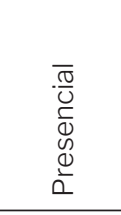 & \\
\hline $\begin{array}{l}\text { Número de } \\
\text { programas } \\
\text { en Colombia }\end{array}$ & 60 & 74 & 1333 & 58 & 278 & 2680 & 57 & 365 & 5166 & 10071 \\
\hline $\begin{array}{l}\text { Número de } \\
\text { programas } \\
\text { en Bogotá }\end{array}$ & 14 & 31 & 481 & 17 & 84 & 597 & 30 & 106 & 1103 & 2463 \\
\hline $\begin{array}{l}\text { Porcentaje } \\
\text { de progra- } \\
\text { mas en } \\
\text { Bogotá }\end{array}$ & 23.30 & 41.90 & 36.10 & 29.30 & 30.20 & 22.30 & 29 & 21.40 & $22,20 \%$ & $24,46 \%$ \\
\hline
\end{tabular}

Fuente: Elaboración propia.

Las tendencias que muestra la tabla 2.3 son las siguientes:

- En la formación en nivel técnico profesional, las dos modalidades a distancia suman menos de $10 \%$ de la oferta de programas en Colombia y $65.2 \%$ de este $10 \%$ se concentra en instituciones afincadas en Bogotá.

- Cerca de 15 \% de los programas de formación tecnológica pertenece a las dos modalidades de educación a distancia y $59.5 \%$ de este $15 \%$ se concentra en instituciones afincadas en Bogotá.

- En el caso de la formación profesional universitaria, los programas a distancia virtual y a distancia tradicional juntos suman $8 \%$ de la oferta en Colombia, y de este $8 \%$ 81.7 \% se concentra en instituciones afincadas en Bogotá.

Estos puntos parecen responder a lo que muestran los datos del DANE sobre una concentración de mayor número de pobladores y de actividades económicas en Bogotá, donde también tiene sede la mayor parte de las instituciones de educación superior. Cabe averiguar si, a pesar de tener sede en Bogotá o en otras grandes ciudades, las instituciones con ofertas virtuales y a distancia han logrado dar acceso a educación superior y, en qué nivel 
de calidad, a estudiantes de zonas rurales de diferentes partes del país y de regiones con las cuales hay menos acceso tradicional de vías, comunicaciones y comercio.

Por otra parte, es importante anotar que la cobertura de programas virtuales ha crecido gracias al aumento de cobertura educativa en Bogotá, que desde el inicio de esta medición concentra la mayor oferta de este tipo de programas; de 2002 a 2009, aumentó la cobertura de programas de educación superior de 55 a $75 \%$. Sin embargo, el aumento en el resto de país fue apenas de 17.70 a 23.01 \% (Institución Universitaria Politécnico Grancolombiano, inédito) (figura 2.3).

Figura 2.3. Cambio en el tiempo del porcentaje aportado por Bogotá y por otras regiones al número de estudiantes en educación superior.

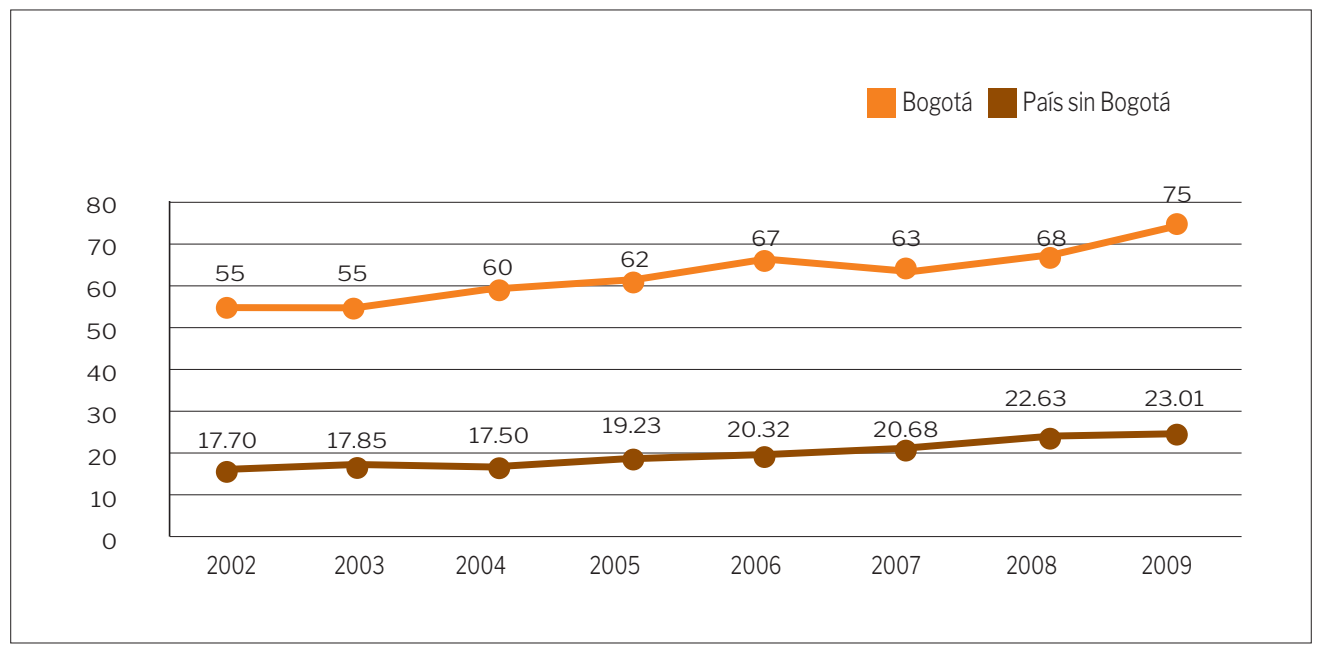

Fuente: DANE (2011)

El interrogante que ahora puede plantearse es si la oferta y su crecimiento en el país son coherentes con el aumento de su población entre 17 y 21 años. El aumento de la cobertura a estudiantes es general en todo el país de 2002 a 2009, pero buena parte de esta es producto de una extensión de la cobertura en Bogotá, que a veces parece aumentar a costa del crecimiento a un ritmo menor de la cobertura en otros lugares del país.

Del mismo modo, entre 2002 y 2009, aumentó la población total cubierta por los programas de educación superior. Este crecimiento es más del doble en Bogotá en este periodo, mientras que en el resto del país es de solo $40 \%$. Esto concuerda con un tercer dato: el porcentaje de población cubierta que aporta Bogotá crece casi de manera constante desde 2002, cuando es $38.69 \%$, hasta 2009, cuando es 49.41 \% (figura 2.4). 
Figura 2.4. Número de estudiantes que son cubiertos por la educación superior en Bogotá y en otras regiones de 2002 a 2009.

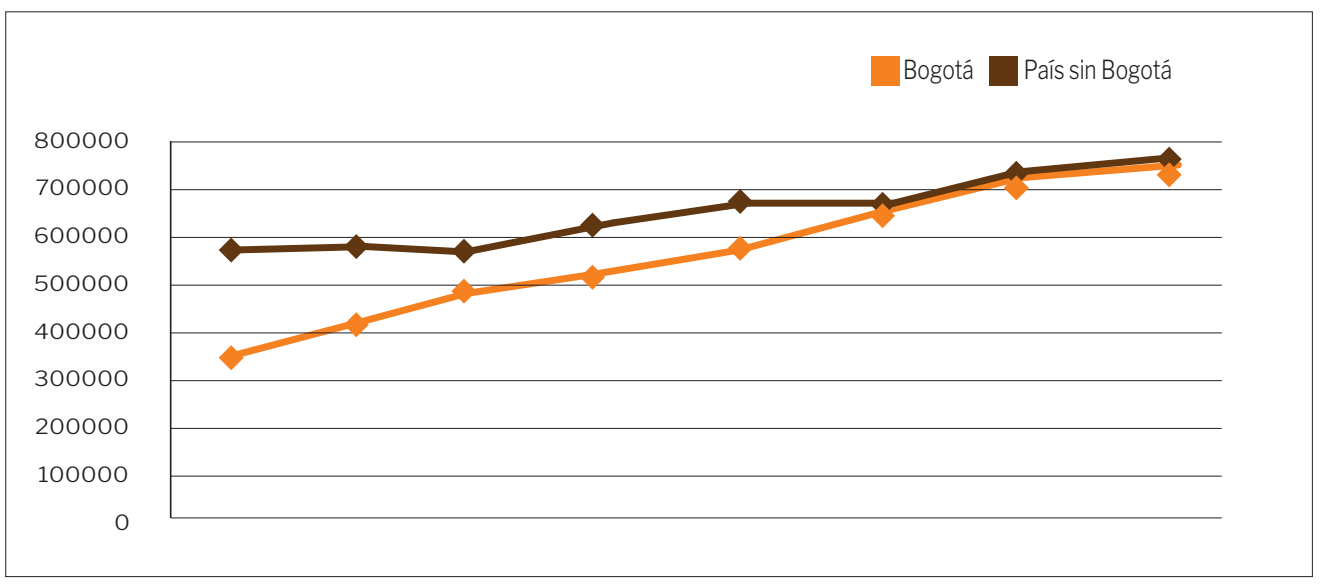

Fuente: DANE (2011).

En términos generales, se observa que, en relación con la población de jóvenes entre 17 y 21 años en Bogotá, la oferta de programas es coherente. No obstante, si se compara la tendencia al aumento de la cobertura y de los estudiantes, se evidencia una gran brecha entre Bogotá y el resto del país (Institución Universitaria Politécnico Grancolombiano, inédito). Es pertinente saber qué se ha hecho en años más recientes con respecto a esta cobertura y qué se ha logrado respecto de las mejoras del desempeño y del aprendizaje comparados de los estudiantes de zonas rurales y de grandes urbes colombianas.

\section{Logros de estudiantes colombianos en zonas rurales y urbanas que po- drían acceder a la educación superior}

Además del anterior estudio y análisis, existen otras evidencias que muestran diferencias entre los estudiantes de procedencia urbana o rural en su logro educativo o en función de factores socioeconómicos o socioculturales de los estudiantes. En ese sentido, los resultados de las pruebas Icfes-Saber 11, analizadas en su curso histórico de 2006 a 2010, muestran lo siguiente:

- Hay una tendencia a que los estudiantes de colegios o escuelas oficiales rurales tengan menor desempeño en el promedio de pruebas genéricas que se aplicaron de 2006 a 2010. Esta diferencia no se da entre los colegios rurales y los colegios urbanos privados. 
- No se observan diferencia entre los estudiante rurales y urbanos oficiales en matemáticas, pero sí una tendencia negativa en los rurales desde 2007, y un aumento en los urbanos de un punto en 2010 en calendario A. Los colegios privados de ambas zonas tienen mayor variabilidad que los oficiales.

- Se observan mayores promedios en los colegios urbanos oficiales que en sus pares rurales en la prueba de lenguaje en calendario A y diferencias de promedios entre estas zonas en calendario A. Los colegios privados de ambas zonas muestran promedios más altos y tienen mayor variabilidad en sus puntajes que los oficiales.

- La misma tendencia del punto anterior en los colegios rurales y urbanos oficiales en pruebas de ciencias naturales y ciencias sociales.

- Los colegios oficiales rurales registran los menores promedios, seguidos de los oficiales urbanos. Esta desventaja tiende a ampliarse en matemáticas, a mantenerse en ciencias naturales y ciencias sociales y a disminuir en lenguaje.

Asimismo, el reporte de los resultados de las pruebas PISA-OCDE 2012 muestra dos resultados interesantes en el mismo sentido:

- Los estudiantes de Bogotá, Medellín y Manizales tienen puntajes más altos que los estudiantes evaluados en otras regiones del país en las pruebas de lenguaje, ciencias y matemáticas que se aplicaron en esta evaluación.

- Las puntuaciones en las pruebas tienden a ser más altas en los estudiantes que residen en ciudades grandes, comparadas con los estudiantes de otras zonas o de escuelas rurales.

- Existe correlación entre el tamaño de la matricula (cobertura de educación a los estudiantes de la región) y las puntuaciones en las zonas de estudiantes de 15 y 16 años que presentaron las pruebas.

Los mayores logros de estudiantes, reflejados en pruebas externas, siguen concentrados en regiones del país que registran mayor población, más vías de acceso y comunicación y mejores indicadores de actividad económica (DANE, 2011). Los logros por áreas muestran una tendencia que se refleja más recientemente en evaluaciones internacionales: mayores brechas en matemáticas, brechas en el mismo nivel en ciencias y menores en lenguaje, al parecer por intervenciones y formas de capacitación y profesionalización de docentes que se vienen dando en el país en las últimas décadas (MinEducación, 2017).
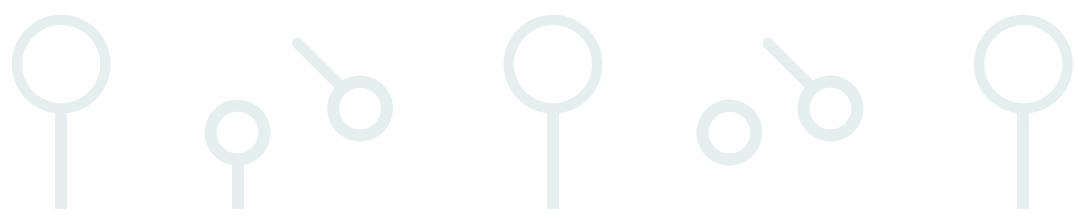
Comunicación, redes, aprendizaje y desarrollo institucional y social

\section{Discusiones}

Los hallazgos de estos años iniciales de implementación de programas virtuales en educación superior en Colombia muestran la siguiente prospectiva para los años siguientes:

- Tratar de romper la concentración de ofertas en Bogotá y en las regiones centrales de Colombia, para llegar a estudiantes de diferentes regiones, incluso desde universidades con sede principal en tales regiones centrales a través de las modalidades o metodologías a distancia tradicional y virtual. Resulta importante indagar sobre si en tiempos más recientes se ha logrado generar oportunidades de acceso a la educación superior de calidad en diferentes regiones de Colombia, donde el panorama inicial muestra un acceso a un ritmo más lento a la educación superior de los jóvenes que aquel que se daba en Bogotá (DANE, 2011).

- Estudiar la incidencia de modalidades o metodologías virtuales o a distancia tradicional sobre el acceso a educación superior con calidad en estudiantes de todas las regiones colombianas, contemplando en esta línea de estudio múltiples factores de los estudiantes, de los programas y de las instituciones, para definir qué puntos tienen incidencia en su éxito académico, en su adquisición de habilidades y competencias e, incluso, en su permanencia y deserción (Arias-Velandia, 2015; Areth, Castro-Martínez y Rodríguez, 2015; Colomina, Onrubia y Rochera, 2001).

En general, los resultados de este estudio muestran una tendencia fuerte de concentración en grandes conglomerados urbanos colombianos de las instituciones con ofertas de educación superior (en diferentes modalidades), de la cobertura a estudiantes y de las mayores calificaciones de logro de aprendizaje de estos. Por esto, es necesario acceder a información que muestre con mayor detalle el alcance a diferentes poblaciones de las modalidades virtuales y a distancia (considerando que los estudiantes de estas modalidades no necesariamente están ubicados en el mismo lugar de residencia física de los programas que toman), de las posibilidades de acceso a estos, tanto en infraestructuras físicas y tecnológicas como en la tendencia de recursos culturales, que les permitan un acceso y permanencia exitosos en educación superior (Silvio, 2004; Hederich, 2007).

Es importante destacar que parte de esta desventaja se presentaba ya en las zonas rurales colombianas. Como mostraron nuestros resultados, la diferencia de acceso a educación, incluso desde la básica secundaria, era más pronunciada en departamentos con mayor población, con más vías de acceso y comunicación y con mejores indicadores económicas, lo cual muestra que las diferencias de acceso a la educación superior no eran solo entre regiones, sino dentro de una misma región en sus grandes urbes y sus espacios rurales. 
Esto coincide con la tesis de Fujita, Krugman y Venables (1999), según la cual el espacio geográfico con mayores oportunidades y dinámicas tiende a seguir acumulando dichas oportunidades y dinámicas, por el hecho de construir las estructuras necesarias de participación de los bienes materiales y culturales. Se trata, entonces, de círculos de dinámicas que se alimentan a sí mismas y por lo cual es difícil salir de estas tendencias (Flórez, Castro y Arias, 2009). Coincide esto también con lo expuesto desde las obras de Romero (2001) y Colmenares (1987), al mostrar la tradicional brecha entre los mundos de campo y ciudad en el desarrollo histórico de América Latina. Ambas dinámicas se reflejaron también en los resultados de las pruebas externas nacionales e internacionales, en las cuales los estudiantes de fuera de los grandes núcleos urbanos tienden a tener menores puntajes que sus pares en las grandes ciudades colombianas (Arias-Velandia, Rojas-Benavides y Hernández-Amórtegui, 2014).

Este es entonces el real contexto de desafíos que tienen tanto la educación virtual como la educación a distancia tradicional en Colombia. Hay buenas perspectivas que tal vez se evidencien en seguimientos del comportamiento de acceso, permanencia, logro educativo y trabajo futuro de los estudiantes de educación superior de zonas rurales, a quienes probablemente lleguen más estas ofertas de educación superior no presencial. En diferentes lugares del mundo, la educación virtual repetidamente ha mostrado pocas o ninguna diferencia en los logros de sus estudiantes en comparación con sus compañeros de ofertas equivalentes en educación presencial (Arias-Velandia, 2015; Brownstein, Brownstein y Gerlowski, 2008; Carrol y Burke, 2010; Fujita, Krugman y Venables, 2009; Larson y Sung, 2009; Tutty y Kleine, 2008; Wong y Tantall, 2009), desafíos para enfrentar la permanencia y la deserción de sus estudiantes con potenciales vulnerabilidades (Areth, Castro-Martínez y Rodríguez, 2015) y capacidad para enfrentar algunos desafíos generales a la educación superior, como los que se han planteado en Colombia (Celis, Jiménez y Jaramillo, 2013; Montoya, 2013). Cabe, entonces, lugar al estudio sistemático con datos de años recientes sobre la respuesta a los desafíos planteados en este capítulo.

\section{Referencias}

Arias-Velandia, N. (2015). Logro educativo comparado entre educación superior presencial y virtual en Colombia: algunas tendencias y grandes desafíos. Recuperado de http:// alejandria.poligran.edu.co/bitstream/handle/10823/443/EDUCACION \%20 SUPERIOR \%20PRESENCIAL \%20Y \%20VIRTUAL.pdf?sequence=2\&isAllowed=y

Arias-Velandia, N., Rojas-Benavides, L. y Hernández-Amórtegui, J. S. (2014). Logro en educación superior presencial y virtual: aproximaciones preliminares. UDI Investiga, 4, 30-44. Recuperado de https://www.udelistmo.edu/sites/default/files/revista-udi-no.4-2014vhm.pdf\#page $=30$

Areth Estévez, J., Castro-Martínez, J. y Rodríguez Granobles, H. (2015). La educación virtual en Colombia: exposición de modelos de deserción. Apertura, 7(1), 1-10. Recuperado de http://www.redalyc.org/pdf/688/68838021007.pdf 
Comunicación, redes, aprendizaje y desarrollo institucional y social

Arum, R. y Roksa, J. (2011). Academically adrift: Limited learning on college campuses. Chicago: University of Chicago.

Bogoya, D. (2006). Evaluación educativa en Colombia. Trabajo presentado en Seminario Internacional de Evaluación, Cartagena de Indias, Instituto Colombiano para la Evaluación de la Educación, Ministerio de Educación Nacional.

Brownstein, B., Brownstein, D. y Gerlowski, D. (2008). Web-based vs. face-to-face MBA classes: A comparative assessment study. Journal of College Teaching \& Learning, 5(11), 41-48.

Campbell Collaboration (2006). What is a systematic review? Recuperado de https://www. campbellcollaboration.org/research-resources/writing-a-campbell-systematic-review/ systemic-review.html

Carrol, N. y Burke, M. (2010). Learning effectiveness using different teaching modalities. American Journal of Business Education, 3(12), 65-76.

Celis, M. T., Jiménez, O. A. y Jaramillo, J. F. (2013). ¿Cuál es la brecha de la calidad educativa en Colombia en la educación media y en la superior? En Estudios sobre calidad de la educación en Colombia (pp. 67-90). Bogotá: Instituto Colombiano para la Evaluación de la Educación.

Colmenares, G. (1987). La formación de la economía colonial (1550-1740). En J. A. Ocampo (comp.), Historia económica de Colombia (2. ed., pp. 13-47). Bogotá: Siglo XXI.

Colomina, R., Onrubia, J. y Rochera, M. J. (2001). Interactividad, mecanismos de influencia educativa y construcción de conocimiento en el aula. En C. Coll, J. Palacios y A. S. Marchesi (comps.), Desarrollo psicológico y educación, vol. 2: psicología de la educación escolar (pp. 437 - 458). Madrid: Alianza.

DANE (Departamento Administrativo Nacional de Estadística) (2011). Informe Especial. Censo General 2005. Bogotá: DANE.

Flórez Romero, R., Castro Martínez, J. y Arias Velandia, N. (2009). Comunicación, lenguaje y educación: una mirada desde las teorías de la complejidad. Folios, 30, 25-38. Recuperado de http://www.scielo.org.co/pdf/folios/n30/n30a02.pdf

Fujita, M., Krugman, P. y Venables, A. J. (1999). The spatial economy: Cities, regions, and international trade. Cambridge: MIT Press.

Gibson, J. W. (2008). A comparison of student outcomes and student satisfaction in three MBA human resource management classes based on traditional vs. online learning. Journal of College Teaching and Learning, 5(8), 1-9.

Hederich Martínez, C. (2007). Estilo cognitivo en la dimensión de dependencia-independencia de campo: influencias culturales e implicaciones para la educación. Bogotá: Universidad Pedagógica Nacional.

Hederich Martínez, C. y Camargo, Á. (2000). Estilo cognitivo y logro en el sistema educativo de la ciudad de Bogotá. Bogotá: Instituto para la Investigación Educativa y el Desarrollo Pedagógico.

Hederich Martínez, C., Martínez Bernal, J. y Rincón Camacho, L. (2014). Hacia una educación basada en la evidencia. Revista Colombiana de Educación, 66, 19-54. 
Institución Universitaria Politécnico Grancolombiano (inédito). Oportunidades de trabajo y oferta de educación superior en Colombia. Bogotá: Institución Universitaria Politécnico Grancolombiano.

Kirtman, L. (2009). Online versus in-class courses: An examination of differences in learning outcomes. Issues in Teacher Education, 18(2), 103-116.

Larson, D. K. y Sung, C. H. (2009). Comparing student performance: Online versus blended versus face-to-face. Journal of Asynchronous Learning Networks, 13(1), 31-42.

Lugo, M. T., Vera Rossi, M. y Flood, C. (2004). Educación superior virtual en Argentina: un relevamiento necesario. En La educación superior virtual en América Latina y el Caribe (pp. 51-84). Caracas: Unesco.

MinEducación (Ministerio de Educación Nacional) (2017). Informe nacional de resultados: Colombia en PISA 2015. Bogotá: MinEducación.

Montoya Cardona, D. (2013). Contribución de la educación superior a la equidad de resultados en Colombia. Trabajo presentado en Seminario Internacional de Investigación sobre Calidad de la Educación, Bogotá, Icfes, MinEducación.

Padilla Omiste, Á. (2004). Diagnóstico de la educación superior virtual en Bolivia. En La educación superior virtual en América Latina y el Caribe (pp. 85-112). Caracas: Unesco.

Prensky, M. (2001). Digital natives, digital immigrants part 1. On the horizon, 9(5), 1-6.

Rivera Rivera, K. (2004). La educación superior a distancia en Centroamérica. En La educación superior virtual en América Latina y el Caribe (pp. 145-164). Caracas: Unesco.

Romero, J. L. (2001). La ciudad latinoamericana: continuidad europea y desarrollo autónomo. En Situaciones e ideologías en América Latina (pp. 227-234). Medellín, Colombia: Universidad de Antioquia.

Silvio, J. (2004). Tendencias de la educación superior virtual en América Latina y el Caribe. En La educación superior virtual en América Latina y el Caribe (pp. 5-28). Caracas: Unesco.

Tomasevsky, K. (2005). Los derechos económicos, sociales y culturales: informe preliminar de la relatora especial de las Naciones Unidas para el derecho a la educación. En M. F. Paz (ed.), El derecho a la educación de niños y niñas en situación de desplazamiento y de extrema pobreza en Colombia (pp. 21-51). Bogotá: Due Process of Law Foundation.

Tutty, J. I. y Klein, J. D. (2008). Computer-mediated instruction: A comparison of online and faceto-face collaboration. Educational Technology Research and Development, 56(2), 101-124.

Vianney, J., Torres, P. y Farias, E. (2004). La educación superior a distancia en Brasil. En La educación superior virtual en América Latina y el Caribe (pp. 113-144). Caracas: Unesco.

Wong, L. y Tatnall, A. (2009). The need to balance the blend: Online versus face-to-face teaching in an introductory accounting subject. Journal of Issues in Informing Science and Information Technology, 6, 309-322. 